\title{
Express in-situ measurement of single crystal diamond growth/etching rate in microwave plasma: how to perform multiparametric kinetics study in one working day
}

\author{
$\underline{\text { V.G. Ralchenko }}^{1,2,3}$, V.Yu. Yurov ${ }^{1,2}$, E.V. Bushuev ${ }^{1}$, A.P. Bolshakov ${ }^{1,2}$, E.E. Ashkinazi ${ }^{1,2}$, \\ I.A. Antonova ${ }^{1,2}$, E.V. Zavedeev ${ }^{1}$, A.A. Khomich ${ }^{1,4}$, V.I. Konov ${ }^{1,2}$ \\ ${ }^{1}$ General Physics Institute RAS, Moscow, Russia, vg ralchenko@mail.ru \\ ${ }^{2}$ National Research Nuclear University MEPhI, Moscow, Russia \\ ${ }^{3}$ Harbin Institute of Technology, Harbin, P.R. China \\ ${ }^{4}$ Institute of Radio Engineering and Electronics RAS, Fryazino, Russia
}

The ability to precisely control and stabilize the growth process of single crystal (SC) diamonds by a microwave plasma chemical vapor deposition (MPCVD) is of paramount importance for achieving high uniformity and quality of the produced crystals for high-tech applications. The conventional approach to experimentally optimize the process parameters relays on preparation of numerous SC samples with ex-situ evaluation of growth rate, that is a tedious and time-consuming procedure, often taking several weeks or months. Here we describe a very effective technique to measure in situ with high accuracy the growth rate of SC diamond using a lowcoherence interferometry (LCI) $[1,2]$. The key advantage of the method is a possibility to use only one substrate to deposit consecutively layer-by-layer under variable conditions and determine kinetics for each of them.

\section{Growth}

The epitaxial SC diamond films were deposited in ARDIS-100 MPCVD system [2] on (100)-oriented Ib HPHT diamond substrates in $\mathrm{H}_{2}-\mathrm{CH}_{4}$ gas mixtures in a broad range of $\mathrm{CH}_{4}$ concentrations (1-13\%) and substrate temperatures $T_{\mathrm{s}}\left(750-1150{ }^{\circ} \mathrm{C}\right)$.

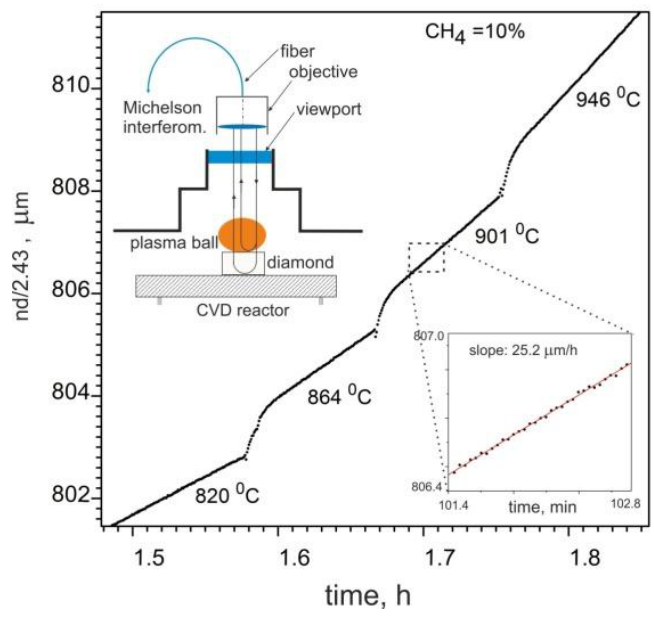

Fig. 1. Evolution of epilayer thickness with time under consecutive rise in steps of substrate temperature at $10 \% \mathrm{CH}_{4}$ content as measured with LCI. The temperature is indicated for each segment. The linear segments correspond to the growth with constant rate. Inset: the optical scheme of LCI measurement of the sample thickness

The beam of the low-coherence infrared radiation from a superluminescent diode (SLD) at $\lambda_{0}=1560 \mathrm{~nm}$ wavelength with bandwidth $\Delta \lambda=30 \mathrm{~nm}$, was directed perpendicularly to diamond sample surface through an objective of Michelson interferometer positioned above the top quartz window of the CVD chamber. The LCI system is able to measure the temporal evolution of optical thickness $n d(t)$ of the etched sample (Fig. 1), where $n$ is refraction index of diamond, and $d$ is the sample thickness. Typically, less than $10 \mathrm{~min}$ growth run was required to get enough data points to evaluate the growth rate with high accuracy at given temperature, then switching to the next $T_{\mathrm{s}}$ value.

Rich growth kinetics is collected in a single experiment by depositing about 60 layers on diamond substrate in different regimes without switch-off the plasma. The plot for temperature dependent growth rate $\mathrm{G}(T)$ is shown in Fig. 2 for different $\mathrm{CH}_{4}$ percentages. The growth rate as high as $82 \mu \mathrm{m} / \mathrm{h}$ is achieved by optimizing the temperature and gas composition without adding nitrogen in the gas. In certain cases, the deposition of a layer as thin as $100 \mathrm{~nm}$ was enough to determine the growth rate. The estimated accuracy limit of $\approx 0.4 \%$ in the $\mathrm{G}(T)$ measured is due the substrate temperature fluctuations and drift. The growth rate is found to follow Arrhenius dependence with activation energy $E_{\mathrm{a}}=11.1 \pm 1.0 \mathrm{kcal} / \mathrm{mol}$ $(\approx 46 \mathrm{~kJ} / \mathrm{mol}$ ), in the middle of the range of $7.4-$ $15.2 \mathrm{kcal} / \mathrm{mol}$ reported by Maeda et al. [3].

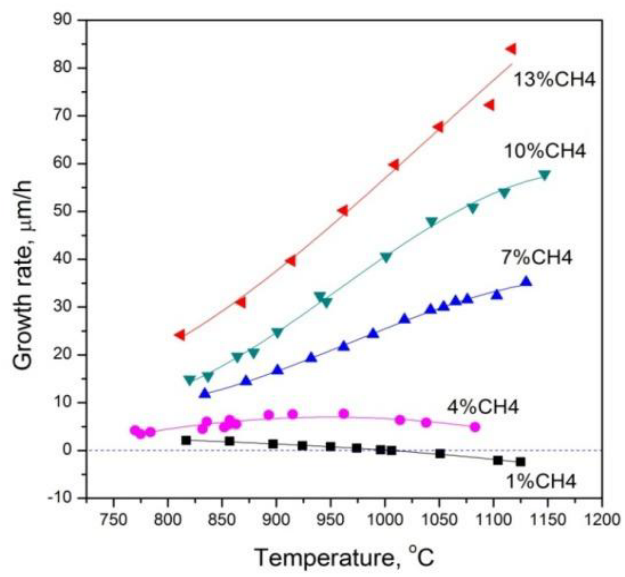

Fig. 2. Dependence of growth rate on substrate temperature $\mathrm{G}(\mathrm{T})$ at different $\mathrm{CH}_{4}$ contents in gas mixture. Note the net etching for $1 \% \mathrm{CH}_{4}$ at $\mathrm{T}_{\mathrm{s}}>1000 \mathrm{~K}$

Raman and photoluminescence (PL) spectra taken on polished cross-section of different layers, confirmed high quality of the material (Fig. 3). The diamond Raman peak width (WFHM) was essentially constant, $\Delta v=2.4 \pm 0.1$ $\mathrm{cm}^{-1}$ through all epilayers $\left(\Delta v=2.3 \mathrm{~cm}^{-1}\right.$ was measured for a reference sample, natural IIa diamond), and only a 
small contamination of Si impurity detected by PL ( $\mathrm{SiV}$ center at $738 \mathrm{~nm}$ ).

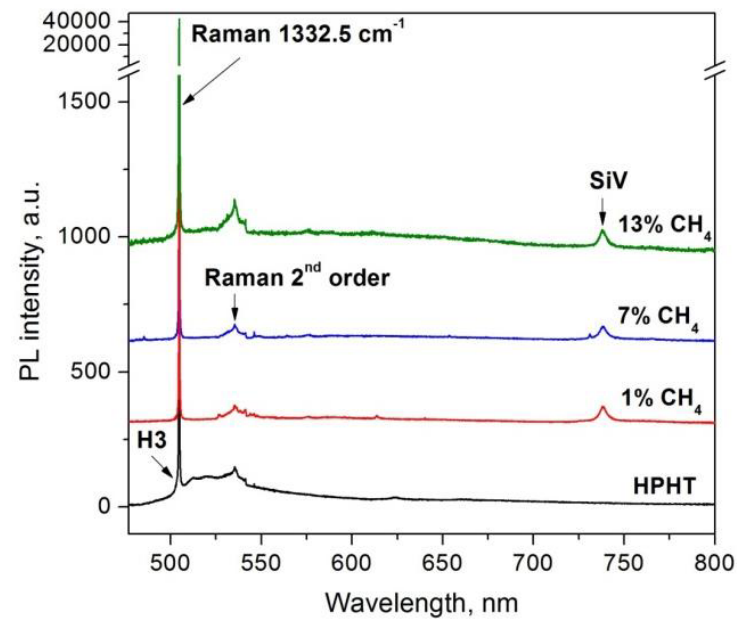

Fig. 3. PL spectra for diamond layers grown at $1 \%, 7 \%$ and $13 \% \mathrm{CH}_{4}$ in gas and for HPHT substrate. The spectra are zoomed to enhance the PL features

\section{Etching in hydrogen plasma}

Similarly, the etch rates in pure $\mathrm{H}_{2}$ plasma for two sorts of synthetic diamond, (100)-oriented IIa HPHT diamond, and CVD plates, were studied in situ using LCI. The etching process was performed at pressure $p=130$ Torr, flow rate of $450 \mathrm{sccm}$, and substrate temperatures $T_{\mathrm{s}}=800-1370^{\circ} \mathrm{C}$, the latter being tuned by the microwave power $P$ in the range of $2.9-4.0 \mathrm{~kW}$.

Table. Diamond SC samples for etching: $\theta$ is off-angle for (100) plane, $[\mathrm{N}]$ is nitrogen impurity concentration.

\begin{tabular}{|l|c|c|c|}
\hline $\begin{array}{c}\text { Sample } \\
\#\end{array}$ & $\begin{array}{c}\text { Size, } \\
\mathrm{mm}\end{array}$ & $\begin{array}{c}\theta, \\
\text { degree }^{\circ}\end{array}$ & $\begin{array}{c}{[\mathrm{N}],} \\
\mathrm{ppb}\end{array}$ \\
\hline IIa HPHT & $5 \times 5 \times 0.50$ & 1.1 & 400 \\
\hline CVD & $4.5 \times 5.1 \times 0.47$ & 4.2 & $<50$ \\
\hline
\end{tabular}

The plot for etching rate $E R$ vs $T_{\mathrm{s}}$ for the HPHT substrate, shows an unusual behavior (Fig. 4). A well defined spike in $E R(T)$ at low temperatures of $800-970^{\circ} \mathrm{C}$ is due to enhanced etching rate of a subsurface damaged (SSD) layer formed by polishing procedure. After the entire removal of that SSD layer, "normal" etching rate inherent to bulk material, is established. A similar picture is was observed for CVD diamond etching. The SSD layer thickness $d \approx 0.2 \mu \mathrm{m}$ and $0.7 \mu \mathrm{m}$ was estimated from the $E R(T)$ plots for IIa HPHT and CVD substrate, respectively.

Assuming exponential temperature dependence for etch rate $E R(T)=A \times \exp \left(-E_{\mathrm{a}} / R T_{\mathrm{s}}\right)$, where $E_{\mathrm{a}}$ is activation energy, and $R$ is universal gas constant, Arrhenius plots for the CVD and IIa HPHT samples were built based on the measured rate (Fig. 5), and the activation energy $E_{\text {a }}$ of $42 \pm 5 \mathrm{\kappa cal} /$ and $32 \pm 4 \mathrm{\kappa cal} / \mathrm{mol}$, was determined for CVD and IIa type HPHT diamond, respectively. The published data [4] for etching of Ib-HPHT diamond in $\mathrm{H}_{2}$ MW plasma with $E_{\mathrm{a}}=55.8 \mathrm{\kappa cal} / \mathrm{mol}$ for temperatures below $950{ }^{\circ} \mathrm{C}$ are also given in Fig. 5 for comparison.

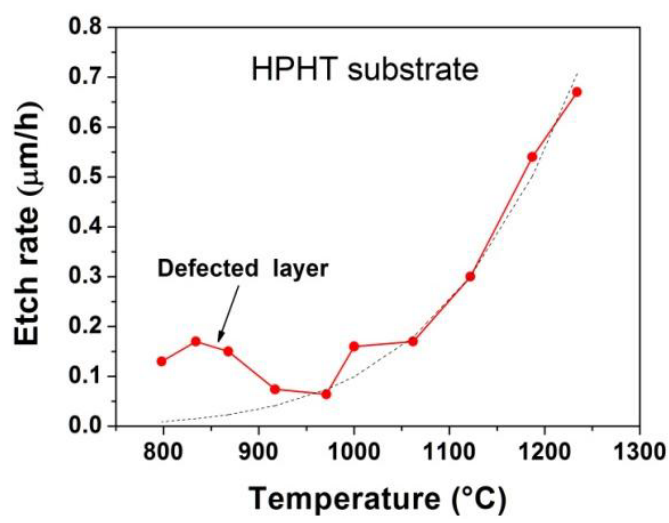

Fig. 4. Etching rates for IIa HPHT sample vs temperature. The dashed lines show Arrhenius approximations for $E R(T)$ at high temperatures (in absence of the defected layer)

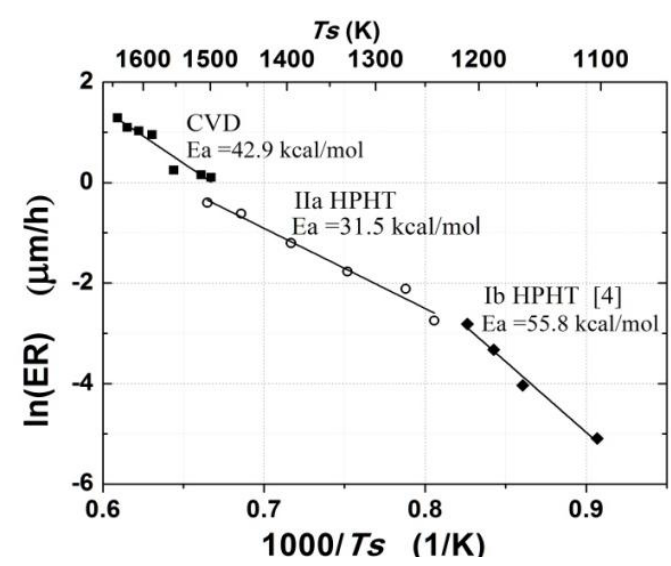

Fig. 5. Arrhenius plots for samples CVD (squares) and Ila HPHT (circles), respectively. The published data for Ib-HPHT diamond [4] (diamonds) are given for comparison

The difference in the activation energy for the CVD and HPHT diamonds can be assigned to a difference in defects abundance in these substrates. Our $E_{\mathrm{a}}$ values are the firstly reported for diamond etching in $\mathrm{H}_{2}$ plasma at temperatures above $1000^{\circ} \mathrm{C}$.

In summary, the in situ LCI technique dramatically reduces the time to collect kinetics data. The full cycle of the measurements, either growth or etching, can be performed during one working day.

This work was supported by the Russian Scientific Foundation, grant No. 14-12-01403-P.

\section{References}

1. Bushuev, E.V., Yurov, V.Yu., et al.. Synthesis of single crystal diamond by microwave plasma assisted chemical vapor deposition with in situ low coherence interferometric control of growth rate // Diam. Relat. Mater. 2016. V. 66. P 83-89.

2. Bushuev, E.V., Yurov, V.Yu., et al. Express in situ measurement of epitaxial CVD diamond film growth kinetics // Diam. Relat. Mater. 2017. V. 72, P. 61-70.

3. Maeda, H., Ohtsubo, K. et al. Determination of diamond [100] and [111] growth rate and formation of highly oriented diamond film by microwave plasma-assisted chemical vapor deposition // J. Mater. Res. 1995. V. 10. P. 3115-3123.

4. Ivanov, O.A., Muchnikov, A.B. et al. Experimental study of hydrogen plasma etching of (100) single crystal diamond in a MPACVD reactor // Mater. Lett. 2015. V. 151. P. 115-118. 